\section{All medicines have side effects}

\author{
Karel Allegaert, ${ }^{1,2}$ Imti Choonara ${ }^{3}$
}

Antihistamines are a widely used group of medicines. As well as being prescribed, they are available over the counter in many countries. A recent study from France highlighted that each year almost one in three children will receive an antihistamine and that in children aged between 2 and 5 years, almost one in two will receive an antihistamine. ${ }^{1}$ The paper by Vries and Hunsel from the Netherlands highlights reported suspected adverse drug reactions (ADRs) to first-generation and secondgeneration systemic antihistamines. ${ }^{2}$

Skin eruptions and ADRs involving the central nervous system (headache, somnolence, aggression, agitation, hyperactivity and seizures) were the most commonly reported $\mathrm{ADRs}$ to second-generation antihistamines. These ADRs are also the ADRs most frequently associated with firstgeneration antihistamines. Second-generation antihistamines are thought to be associated with fewer side effects. Their findings do not suggest that we should stop using antihistamines in children. They do, however, highlight that medicines we consider to be safe do have side effects.

We know that many side effects are not recognised as such by health professionals. As antihistamines are used for the treatment of allergic reactions, one needs to be aware that skin eruptions are the most commonly reported ADRs to antihistamines. The mechanism of skin eruptions due to antihistamines is unknown. The fact that it occurs with a variety of antihistamines suggests that it is a risk associated with all antihistamines. If therefore any child receiving an antihistamine develops a fixed skin eruption, one needs to consider the possibility that the drug prescribed is responsible.

\section{HOW COMMON ARE ADRs?}

The majority of ADRs are not reported. Additionally, many ADRs are not recognised. It is therefore difficult to accurately

\footnotetext{
${ }^{1}$ Intensive Care and Department of Surgery, Erasmus MC-Sophia Children's Hospital, Rotterdam, The Netherlands; ${ }^{2}$ Department of Development and Regeneration, KU Leuven, Leuven, Belgium; ${ }^{3}$ Academic Division of Child Health, University of Nottingham, Derbyshire Children's Hospital, Derby, UK

Correspondence to Professor Imti Choonara, Academic Unit of Child Health, The Medical School, University of Nottingham, Derbyshire Children's Hospital, Uttoxeter Road, Derby DE22 3NE, UK; imti.choonara@nottingham.ac.uk
}

state how common ADRs in children are. We know that 1 in 10 children in hospital will experience ADRs. ${ }^{3}$ The prevalence of ADRs in the community, however, is uncertain. We know from a regional study in Cuba that at least 1 in 500 children will experience an ADR each year. ${ }^{3}$ This figure, however, is likely to be an underestimate. Certain medicines are more likely to be associated with ADRs. Cytotoxic agents and antiepileptic drugs are commonly associated with ADRs. It is important to recognise, however, that widely used medicines that we consider safe may also be associated with ADRs. The risks and benefits for each medicine need to be carefully considered.

\section{PHARMACEUTICAL COMPANY SPONSORED STUDIES}

Second-generation systemic antihistamines are marketed as being safer than firstgeneration antihistamines. It was of interest that the pharmaceutical company studies of second-generation antihistamines did not describe the ADRs documented by Vries and Hunsel. One of the studies actually reported convulsions in four children receiving levocetirizine. ${ }^{4}$ However, the authors considered that the drug was not responsible. The bias associated with pharmaceutical company studies is well recognised in adults. This bias has been documented mainly in relation to effectiveness. ${ }^{5}$ It is also a problem in relation to toxicity. We are uncertain whether paediatric health professionals are aware of this bias to the same extent.

\section{LOOKING FOR ADRs}

Randomised controlled trials are rightly considered to be the best way of determining whether a drug is effective or not. They are not, however, the best way to evaluate drug toxicity. ${ }^{3}$ Most clinical trials are not sufficiently powered to detect ADRs. Additionally, ADRs are usually poorly reported. Prospective cohort studies and national spontaneous reporting schemes for ADRs are the more effective methods for detecting uncommon ADRs. ${ }^{3}$ Health professionals have a responsibility to report suspected ADRs to the regulatory agencies.

- All drugs may result in ADRs.

- Reporting new and serious suspected ADRs is essential to improve pharmacotherapy.
- Stopping a medication may be as important as starting one.

\section{USING MEDICINES RATIONALLY}

One needs to recognise that all medicines may have ADRs. Additionally, one also needs to be aware that many medicines that are prescribed are not actually needed. There is growing recognition in elderly patients that many long-term medications are not required. There are numerous tools that have been developed to assess whether medicines prescribed to the elderly are appropriate or not. Within paediatrics, only one such tool has been developed-the omission of prescriptions and inappropriate prescriptions (POPI). ${ }^{6}$ This is an important area of research that has been neglected in paediatrics. The rational use of medicines is recognised as being a major problem in low-income countries but there is increasing awareness that it is also a problem in high-income countries. One needs to ask the following questions before prescribing either a new medicine or repeating a prescription for an existing treatment.

- Is a medicine required?

- Is the medicine to be prescribed likely to be effective, in this age group for this condition?

- What are the likely side effects?

- What is the risk/benefit to this individual patient?

- How long is treatment required for?

It is only by asking such questions that one can ensure that patients will receive the optimal treatment.

Funding KA was supported by the Fund for Scientific Research, Flanders (fundamental clinical investigatorship 1800214N) and facilitated by the agency for innovation by Science and Technology in Flanders (IWT, SAFEPEDRUG project IWT/SBO 130033).

Competing interests None declared.

Provenance and peer review Commissioned; internally peer reviewed.

To cite Allegaert K, Choonara I. Arch Dis Child 2016;101:951-952.

Received 7 May 2016

Revised 24 May 2016

Accepted 1 June 2016

Published Online First 23 June 2016

\section{Linked}

http://dx.doi.org/10.1136/archdischild-2015-310315

Arch Dis Child 2016;101:951-952.

doi:10.1136/archdischild-2016-311014

\section{REFERENCES}

1 Bénard-Laribière $A$, Jové J, Lassalle $R$, et al. Drug use in French children: a population-based study. Arch Dis Child 2015;100:960-5. 


\section{Editorial}

2 de Vries TW, van Hunsel F. Adverse drug reactions of systematic antihistamines in children in the Netherlands. Arch Dis Child 2016;101: $968-70$.

3 Choonara I. Aspects of clinical pharmacology in children: pharmacovigilance and safety. Eur J Pediatr 2013;172:577-80.
4 Simons FER. on behalf of the Early Prevention of Asthma in Atopic Children (EPAAC) Study Group. Safety of levocetrizine treatment in young atopic children: an 18-month study. Pediatr Allergy Immunol 2007;18:535-42.

5 Lexchin J, Bero LA, Djulbegovic B, et al. Pharmaceutical industry sponsorship and research outcome and quality: systematic review. BMJ 2003;326:1167.

6 Prot-Labarthe S, Weil T, Angoulvant F, et al. POP (Pediatrics: Omission of Prescriptions and

Inappropriate prescriptions): development of a tool to identify inappropriate prescribing. PLOS ONE 2014;9:e101171. 